\title{
Dietary patterns and risk of oesophageal squamous cell carcinoma: a case-control study
}

\author{
Bahareh Hajizadeh ${ }^{1,2, *}$, Bahram Rashidkhani ${ }^{2}$, Anahita $\mathrm{H} \mathrm{Rad}^{2}$, Seyed M Moasheri ${ }^{3}$ \\ and Hoijatollah Saboori ${ }^{1}$ \\ ${ }^{1}$ Department of Radiation Oncology, Kurdistan University of Medical Sciences, Iran: ${ }^{2}$ Department of Community \\ Nutrition, School of Nutrition and Food Science Shaheed Beheshti University of Medical Sciences, PO Box \\ 19826-19573, Tehran, Iran: ${ }^{3}$ Mashhad University of Medical Sciences, Tehran, Iran
}

Submitted 21 January 2009: Accepted 6 January 2010: First published online 1 March 2010

\begin{abstract}
Objective: We conducted a hospital-based, case-control study to examine the association between dietary patterns and the risk of oesophageal squamous cell carcinoma in Iran.

Design: A total of forty-seven patients with oesophageal squamous cell carcinoma and ninety-six controls underwent face-to-face interviews. Factor analysis was used to detect dietary patterns. Multivariate logistic regression was used to estimate OR and $95 \%$ CI.

Results: We defined two major dietary patterns in this population: 'healthy diet' (high in vegetables, nuts, fruits, low-fat dairy and fish) and 'western diet' (high in solid oil, sugar, sweets, tea, eggs, pickles and processed meat). Both healthy and western pattern scores were divided into two categories (based on medians). Higher healthy pattern scores were significantly associated with decreased risk of oesophageal squamous cell carcinoma (high: second median $v$. low: first median, $\mathrm{OR}=0 \cdot 17,95 \%$ CI $0 \cdot 19,0 \cdot 98)$. An increased risk of oesophageal squamous cell carcinoma was observed with the western pattern (high: second median $v$. low: first median, $\mathrm{OR}=10 \cdot 13,95 \%$ CI $8 \cdot 45,43 \cdot 68$ ).

Keywords Conclusions: The results of the present study suggested that diet might be asso- Oesophageal squamous cell carcinoma ciated with oesophageal carcinoma.

Factor analysis
\end{abstract}

Squamous cell carcinoma of the oesophagus is the sixth most common cancer in the world. However, it ranks fourth in developing countries and occurs more frequently in certain regions of the world, such as Iran, China, South Africa and France. The latest epidemiological report indicated the highest incidence rate of this cancer in $\operatorname{Iran}^{(1)}$.

Oesophageal squamous cell carcinoma is strongly associated with tobacco use and alcohol consumption, but various aspects of diet have a role in increasing the risk $^{(2)}$. A number of studies have tried to explore the relationship between diet and the risk of oesophageal cancer, but mainly focused on individual food items ${ }^{(3,4)}$.

Investigating patterns of dietary intake, rather than focusing on individual dietary components, has recently been recommended as an appropriate approach in nutritional epidemiology ${ }^{(5)}$. Factor analysis is a data-driven (a posteriori) method that describes existing eating patterns of a certain population. It is based on the assumption that betweenperson variations can, in part, be explained by the underlying unmeasured variables (factors). Correlations between the consumption of different food items in the study population are used to identify these underlying factors or patterns, and individuals are then ranked in terms of how closely they conform to the total pattern ${ }^{(6)}$. The current data in the area of dietary pattern and cancers have been drawn from epidemiological studies conducted in the Western world. Nearly two-thirds of these studies were conducted in either Europe or North America, so the results are representative of only a fraction of the global population. There is a lack of published data in developing countries. Epidemiological studies in these countries could substantiate existing evidence, but they could also provide valuable new information.

To assess the role of diet in the aetiology of squamous cell carcinoma of the oesophagus, as well as to provide a dietary guideline that has an important public health implication, we tried to identify major dietary patterns in our study population. In addition, we studied their associations with the risk of oesophageal squamous cell carcinoma in a hospital-based case-control study in a province of Iran with a high prevalence of this cancer.

\section{Materials and methods}

The present study was conducted in Kurdistan Province in Iran. Cases were patients aged $40-75$ years, admitted to 
the major general hospitals in the area under study with incident, histologically confirmed cases of squamous cell carcinoma of the oesophagus, diagnosed not before 6 months of the interview, with no history of cancer of other sites. Controls were patients who were admitted to the same hospital as the cases for a wide spectrum of acute, non-neoplastic conditions that were not related to smoking, alcohol consumption and long-term modification of diet. Controls were frequency-matched with cases on age (5-year groups) and sex.

In total, fifty patients with oesophageal squamous cell carcinoma and 100 controls underwent face-to-face interviews by specifically trained professional interviewers. We excluded two patients from the analysis because their log scales of total energy intake were either $<3$ or $>3$ sD from the mean, indicating errors in their responses to the dietary questions. We further excluded three patients due to missing BMI and two patients due to poor responses with regard to dietary questions. Finally, the data for forty-seven cases with oesophageal squamous cell carcinoma and ninety-six controls remained for analysis.

\section{Dietary assessment}

Usual dietary intake was assessed by means of a 125-item semi-quantitative FFQ. This FFQ had good relative validity and reproducibility for several nutrient intakes among Iranian (Tehranian) adults and appeared to be an acceptable tool for assessing dietary intakes in this population ${ }^{(7)}$. Age-adjusted and deattenuated energy intake correlation coefficients between $24 \mathrm{~h}$ dietary recalls (repeated for 12 months) and the FFQ ranged from $0 \cdot 14$ (vitamin A) to $0 \cdot 71$ (phosphorus) in men (mean $r=0.53$ ) and from 0.11 ( $\beta$-carotene) to $0 \cdot 60$ (fibre) in women (mean $r=0 \cdot 39$ ). Energy-adjusted reproducibility coefficients varied from 0.41 (monounsaturated fat) to 0.79 (protein) in men (mean $r=0.59$ ) and from 0.41 (vitamin A) to 0.74 (saturated fat) in women (mean $r=0 \cdot 60$ ). The FFQ consisted of a list of foods with a standard serving size commonly consumed by Iranians and was used to evaluate dietary habits of the controls 1 year before interview and the cases 1 year before diagnosis. All questionnaires were administered by trained dietitians. Participants were asked to report their frequency of consumption of a given serving of each food item on a daily (e.g. bread), weekly (e.g. rice or meat) or monthly (e.g. fish) basis. We transformed the data into average monthly intake for every food item by assuming that 1 month is equal to $30 \cdot 5 \mathrm{~d}$. Alcohol consumption was not answered by our participants due to cultural barriers; therefore, it was not included in the analysis.

To reduce the complexity of the data, we grouped the individual items into twenty-six separate food groups as shown in the Appendix. Grouping was based on the similarity of nutrient profiles or their association with cancer and is similar to what was used in earlier studies ${ }^{(3,8)}$.

In addition to the dietary history, the questions covered demographic characteristics, gastro-oesophageal reflux symptoms (heartburn and regurgitation), anthropometric measures, smoking, history of medication use, physical activity and occupational history. The consumption of opium was not answered by our participants due to their cultural beliefs and was not included in the analysis.

\section{Statistical methods}

We used the principal components method for factor analysis of the Statistical Package for the Social Sciences statistical software package version 14 (SPSS Inc., Chicago, IL, USA) to identify potential dietary patterns. Using the correlation matrix of the twenty-six food groups, the orthogonal varimax rotation method was applied to achieve a simpler structure facilitating interpretation. Although factors with eigenvalues (a measure of the amount of variance that is accounted for) $>1 \cdot 0$ indicate that the factor describes more variability in the data than the average variability for any individual item within the factor, the selection of factors was based on interpretability and scree $\mathrm{plot}^{(9)}$. In fact, the scree plot showed a clear break, and we selected two factors with eigenvalues $>1 \cdot 9$. Factor loadings are correlation coefficients between food groups and dietary patterns. A positive loading in a factor indicates a direct association with the factor, whereas a negative loading indicates that the food group is inversely associated with the factor. Factor scores were then calculated for each study participant for two dietary patterns (healthy and western patterns). Both healthy and western pattern scores were divided into two categories (based on medians). We used $\chi^{2}$ tests to check the differences of distribution of categorical variables (e.g. symptomatic gastro-oesophageal reflux and smoking), and analysis of variance tests to check the differences of distribution of continuous variables (e.g. BMI) across dietary pattern score categories. Unconditional logistic regression was used to estimate OR with 95\% CI. Age, sex, years of education, symptomatic gastro-oesophageal reflux, BMI, smoking (a combination of smoking status and pack/year), physical activity and total energy intake were considered as potential confounders and were also included in the models. All the reported $P$-values are two-sided.

\section{Etbical considerations}

The present study was approved by all regional ethics committees in Iran. A written informed consent was obtained from the individual before the interview.

\section{Results}

Table 1 shows the distribution of forty-seven cases of oesophageal squamous cell carcinoma and ninety-six controls according to the selected variables. By design, age and sex distributions were similar in cases and controls. Cases had higher tobacco consumption and symptomatic gastro-oesophageal reflux. Controls had higher BMI and years of education. 
Table 1 Characteristics of the patients in a case-control study of oesophageal squamous cell carcinoma in Iran

\begin{tabular}{lcc}
\hline & Oesophageal squamous cell carcinoma & Controls \\
\hline$n$ & 47 & 96 \\
Age (years) & $58(10 \cdot 1)^{\star}$ & $58(10 \cdot 4)^{\star}$ \\
Female (\%) & $29(62)$ & $58(60)$ \\
Symptomatic gastro-oesophageal refluxt (\%) & $16(34)$ & $9(9)$ \\
BMl (kg/m ${ }^{2}$ ) & $20 \cdot 4(3 \cdot 2)^{*}$ & $25 \cdot 3(4 \cdot 2)^{\star}$ \\
Smoking history & & $68(71)$ \\
Never smoker (\%) & $28(60)$ & $7(7)$ \\
Ex-smoker, pack year $<10(\%)$ & $6(13)$ & $12(13)$ \\
Ex-smoker, pack year $\geq 10(\%)$ & $4(8)$ & $5(5)$ \\
Current smoker, pack year $<20(\%)$ & $3(6)$ & $4(4)$ \\
Current smoker, pack year $\geq 20(\%)$ & $6(13)$ & $80(83)$ \\
Years of education & $42(89)$ & $12(13)$ \\
$0(\%)$ & $5(11)$ & $4(4)$ \\
$1-5(\%)$ & - & \\
$5-11(\%)$ & & $(4)$ \\
\hline
\end{tabular}

*indicates mean (SD); otherwise data are presented as $n(\%)$.

tPatients who present with the typical gastro-oesophageal reflux disease (GERD) symptoms of heartburn and regurgitation.

Table 2 Factor-loading matrix for the food groups that represent the two major dietary patterns derived from the FFQ*

\begin{tabular}{|c|c|c|}
\hline Food group & Healthy diet & Western diet \\
\hline Vegetables & $0 \cdot 834$ & - \\
\hline Nuts & 0.733 & - \\
\hline Fruit & 0.707 & - \\
\hline Tomato & 0.622 & - \\
\hline Low-fat dairy & 0.558 & - \\
\hline Fish & 0.524 & - \\
\hline Juice & 0.490 & - \\
\hline Legumes & 0.337 & - \\
\hline Whole grains & 0.332 & -0.279 \\
\hline Red meat & 0.322 & 0.219 \\
\hline Poultry & 0.288 & - \\
\hline Liquid oil & 0.282 & -0.489 \\
\hline Solid oil & -0.268 & 0.704 \\
\hline Sugar & - & 0.631 \\
\hline Sweets & 0.490 & 0.574 \\
\hline Tea & - & 0.486 \\
\hline Egg & 0.385 & 0.465 \\
\hline Pickle & 0.331 & 0.427 \\
\hline Processed meat & - & 0.411 \\
\hline Refined grains & - & 0.393 \\
\hline Soft drinks & - & 0.384 \\
\hline Animal butter & - & 0.374 \\
\hline Fried potatoes & - & 0.353 \\
\hline Snacks & $0 \cdot 225$ & 0.339 \\
\hline Cooked potatoes & - & 0.294 \\
\hline High-fat dairy & - & - \\
\hline Proportion of variability (\%) & 16 & 12 \\
\hline
\end{tabular}

${ }^{*}$ Absolute values of $<0.2$ are not shown in the table for simplicity.

Table 2 shows correlations between individual food items and the pattern factors with eigenvalues $>1 \cdot 9$. The first dietary pattern, accounting for $16 \%$ of the variability, was characterised by high intake of foods generally thought to be healthy (including vegetables, nuts, fruits, tomato, low-fat dairy, fish and juice) and was labelled as 'healthy diet'. The second dietary pattern, labelled as 'western diet', was characterised by high consumption of solid oil, sugar, sweets, tea, eggs, pickle and processed meat.

Table 3 shows the distribution of some established risk factors for oesophageal squamous cell carcinoma according to tertiles of the two dietary pattern scores. In general, there were no obvious differences of distribution of symptomatic gastro-oesophageal reflux and smoking across any of the two dietary patterns. Compared to participants in the lowest category of healthy diet scores, those in the highest category had higher BMI and total energy intake. Compared to participants in the lowest category of western diet scores, participants in the highest category had lower BMI and higher total energy intake.

The OR and their 95\% CI for squamous cell carcinoma of the oesophagus by the median of dietary pattern scores are shown in Table 4. After controlling for the potential confounding effects of age, sex, symptomatic gastrooesophageal reflux, years of education, BMI, smoking, physical activity and total energy intake, the healthy diet pattern tended to decrease the risk of oesophageal squamous cell carcinoma (high: second median $v$. low: first median, $\mathrm{OR}=0 \cdot 17,95 \% \mathrm{CI} 0 \cdot 19,0 \cdot 98)$. An increased risk of oesophageal squamous cell carcinoma was observed with the western dietary pattern (high: second median $v$. low: first median, $\mathrm{OR}=10 \cdot 13,95 \% \mathrm{CI} 8 \cdot 45,43 \cdot 68)$.

\section{Discussion}

By using the factor analysis technique, two dietary patterns were identified among the Iranian Kurdish population. These patterns were characterised by high vegetables, nuts, fruits, tomato, low-fat dairy and fish intake and high solid oil, sugar, sweets, tea, eggs, pickle, and processed meat intake. The first pattern was significantly associated with a lower risk of oesophageal squamous cell carcinoma. Participants who scored high for the second pattern tended to have significant elevated risks for oesophageal squamous cell carcinoma.

The strength of our study is the high participation rate (more than 90\%). The present study was carried out in a province with a very high point prevalence of oesophageal 
Table 3 Selected subjects' characteristics by tertiles of dietary pattern scores*

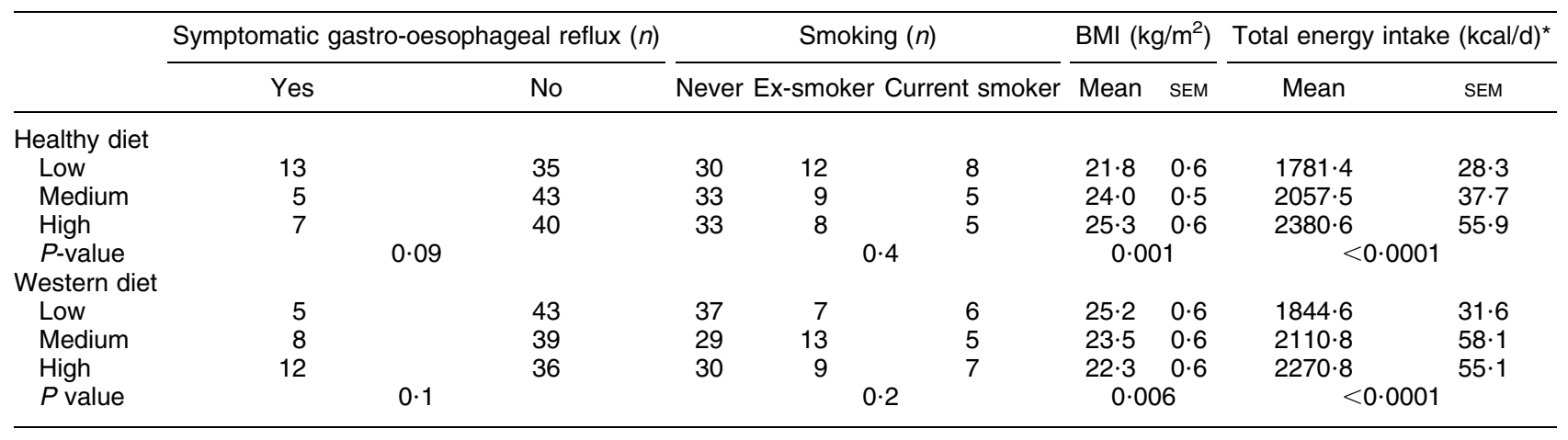

${ }^{*} 1 \mathrm{kcal}=4 \cdot 184 \mathrm{~kJ}$.

Table 4 Oesophageal squamous cell carcinoma risk associated with dietary patterns*

\begin{tabular}{lcccccc}
\hline & Controls $(n)$ & Cases $(n)$ & Crude OR & $95 \% \mathrm{Cl}$ & Adjusted OR & $95 \% \mathrm{Cl}$ \\
\hline Healthy diet & & & & & $1 \cdot 00$ \\
$\quad$ Low & 40 & 33 & 0.29 & $0 \cdot 14,0.62$ & $0 \cdot 17$ & $0 \cdot 19,0.98$ \\
$\quad$ High & 56 & 14 & & $1 \cdot 00$ & $1 \cdot 00$ \\
$\begin{array}{l}\text { Western diet } \\
\text { Low }\end{array}$ & 62 & 9 & 7.94 & $3 \cdot 41,18.33$ & $10 \cdot 13$ & $8 \cdot 45,43 \cdot 68$ \\
High & 34 & 38 & & & & \\
\hline
\end{tabular}

${ }^{*}$ Age, sex, symptomatic gastro-oesophageal reflux, years of education, BMI, smoking, physical activity and total energy intake were also included in the regression models as covariates.

cancer. Studies in developing countries can provide unique opportunities to test the association between diet and cancer $^{(10)}$. Where economic resources are severely restricted, food intake is strongly linked to income so that even small economic differences are directly reflected in diet. This linkage would tend to increase between-person variations. In addition, their socio-economic backgrounds are quite different from the majority of people in the Western world. There is a wide range of differences in demographic-related factors; mainly the status of women, work participation, family size, parity, preferences for male offspring, autonomy and access to health-care systems, which can play a vital roles in influencing the outcome.

However, before the implications of our findings are considered, it is necessary to consider potential biases. First, dietary measurement error may lead to underestimation of results. In addition, the measurement error in confounders will lead to residual confounding ${ }^{(11)}$. Although we cannot entirely rule out residual confounding due to imprecise measurement of important covariates, it is unlikely that errors in measuring the covariates would be so extreme because the crude and multivariable results were essentially the same. Second, due to the cross-sectional design of the study, one cannot infer causality. Therefore, our findings need to be confirmed in future prospective studies. Third, several subjective or arbitrary decisions in the use of factor analysis need to be considered. The investigator is forced to pre-specify the number of factors. Although eigenvalues, scree plots and interpretability are used to guide the investigator in determining the best factor solution, ultimately such a decision is subjective ${ }^{(12)}$. Fourth, because of the small number of participants, associations with wide CI for the western dietary pattern were found.

Some studies have assessed the association between dietary patterns and squamous cell carcinoma ${ }^{(3,13)}$. Bahmanyar and Weimin ${ }^{(3)}$ investigated the association between dietary patterns identified by factor analysis and the risk of oesophageal squamous cell carcinoma in a population-based, case-control study including forty-seven female and 118 male Swedish oesophageal squamous cell carcinoma cases.

The first two major dietary patterns identified in our data (healthy and western) are similar to those identified in the Swedish study ${ }^{(3)}$, using the same method (factor analysis). Although dietary pattern analyses should be interpreted with caution because patterns depend on the geographical and cultural (different dietary preferences) as well as methodological variations (sampling, food grouping, number of variables used in factor analysis, deciding on the number of factors, the rotations employed), two major patterns (healthy and western) were common in the Iranian and Swedish participants. In this Swedish study, there was a significant association between alcohol consumption and oesophageal cancer. The consumption of alcohol and opium was not answered by our participants (due to culture) and therefore were not included in the analysis.

Our study results are generally comparable to those reported by De Stefani et $a l^{(13)}$ that exploratory factor analysis of squamous cell carcinoma of the oesophagus in a 
hospital-based, case-control study in Uruguay, including 234 cases and 468 controls, identified four patterns that were labelled traditional, healthy, high-fat and drinker. The traditional pattern was not associated with the risk of oesophageal carcinoma, whereas the healthy pattern displayed a strong inverse association with this malignancy. Unexpectedly, the high-fat food diet was also marginally protective. On the other hand, the drinker pattern displayed a twofold increase in the risk of oesophageal carcinoma.

Sapkota et $a l^{(14)}$ concluded that the consumption of dairy products was negatively associated with oesophageal cancer. Navarro et $a l^{(15)}$ reported that meat alternatives were associated with a decreased risk of this cancer, while high-fat dairy intake was associated with an increased risk. Most studies in Western populations also found an inverse association between intake of fresh vegetables and fruits, or nutrient intakes derived from these sources, and the risk of oesophageal cancer ${ }^{(3,16-22)}$. Vegetables and fruits are rich in several micronutrients and other food compounds such as carotenoids, vitamins $\mathrm{C}$ and $\mathrm{E}$, fibres, flavonoids and other plant sterols, which display both complementary and overlapping mechanisms of action, including antioxidant effects, binding and dilution of carcinogens and alteration of hormone metabolism $^{(16)}$.

We found that a healthy diet pattern is inversely associated with oesophageal squamous cell carcinoma and the western diet pattern seemed to be associated with oesophageal squamous cell carcinoma. It is reasonable to postulate that the changed dietary habits in populations in recent decades, characterised by high intake of sugar and fat, may partly explain the observed increasing incidence rates of oesophageal carcinomas, although the underlying mechanism is still unclear.

In conclusion, the present findings suggested that a diet characterised by high intake of vegetables, nuts, fruits and fish (labelled as healthy diet) might be negatively associated with the risk of oesophageal squamous cell carcinoma, whereas a diet with high consumption of solid oil, sugar, sweets and processed meat (named western diet) might be positively associated with oesophageal squamous cell carcinoma.

\section{Acknowledgements}

None of the authors had any personal or financial conflicts of interest. The present study was supported by a grant from the National Nutrition and Food Technology Research Institute (NNFTRI) of Iran. B.R. and B.H. designed the study, analysed the data and wrote the manuscript. A.H. helped analyse the data. S.M.M. supervised the study. The authors are grateful to the Kurdistan University of Medical Sciences. The authors thank Drs Saburi, H. Mohammadi and S. Arshiae for their valuable help in conducting the study.

\section{References}

1. Munoz N (1993) Epidemiological aspects of oesophageal cancer. Endoscopy 25, 609-612.

2. Blot WJ, McLaughlin JK \& Fraumeni JF Jr (2006) Esophageal cancer. In Cancer Epidemiology and Prevention, 3th ed., pp. 697-706. [D Schottenfeld and JF Fraumeni Jr, editors]. New York: Oxford University Press.

3. Bahmanyar S \& Weimin Y (2006) Dietary patterns and risk of squamous-cell carcinoma and adenocarcinoma of the esophagus and adenocarcinoma of the gastric carcinoma. Nutr Cancer 54, 171-178.

4. Nyren O \& Adami HO (2002) Esophageal cancer. In Textbook of Cancer Epidemiology, pp. 137-152 [HO Adami, D Hunter and D Trichopoulos, editors]. New York: Oxford University Press.

5. Hu FB, Rimm E, Smith-Warner SA et al. (1999) Reproducibility and validity of dietary patterns assessed with a foodfrequency questionnaire. Am J Clin Nutr 69, 243-249.

6. Gao X, Chen H, Fung TT et al. (2007) Prospective study of dietary pattern and risk of Parkinson disease. Am J Clin Nutr 86, 1486-1494.

7. Mirmiran P, Hosseini Esfahani F, Mehrabi Y et al. (2009) Reliability and relative validity of a food frequency questionnaire for nutrients in the Tehran Lipid and Glucose Study. $J$ Public Health Nutr (Epublication ahead of print version).

8. Rashidkhani B, Ye W, Terry P et al. (2004) Reproducibility and validity of major dietary patterns among Swedish women assessed with a food-frequency questionnaire. J Nutr 134, 1541-1545.

9. Kim JO \& Mueller CW (1978) Factor Analysis: Statistical Methods and Practical Issues. Thousand Oaks, CA: Sage Publications.

10. Willett WC (1998) Nutritional Epidemiology, 2nd ed. New York: Oxford University Press.

11. Fewell Z, Davey Smith G \& Sterne JA (2007) The impact of residual and unmeasured confounding in epidemiologic studies: a simulation study. Am J Epidemiol 166, 646-655.

12. Newby PK, Muller D, Hallfrisch J et al. (2004) Food patterns measured by factor analysis and anthropometric changes in adults. Am J Clin Nutr 80, 504-513.

13. De Stefani E, Boffetta P, Ronco AL et al. (2008) Exploratory factor analysis of squamous cell carcinoma of the esophagus in Uruguay. Nutr Cancer 60, 188-195.

14. Sapkota A, Hsu CC, Zaridze D et al. (2008) Dietary risk factors for squamous cell carcinoma of the upper aerodigestive tract in Central and Eastern Europe. Cancer Causes Control 19, 1161-1170.

15. Navarro SA, Mayne ST, Risch H et al. (2008) Food group intake and risk of subtypes of esophageal and gastric cancer. Int J Cancer 123, 852-860.

16. Lucenteforte E, Garavello W, Bosetti C et al. (2008) Diet diversity and the risk of squamous cell esophageal cancer. Int J Cancer 123, 2397-2400.

17. Kabat GC, Ng SK \& Wynder EL (1993) Tobacco, alcohol intake, and diet in relation to adenocarcinoma of the esophagus and gastric cardia. Cancer Causes Control 4, 123-132.

18. Tzonou A, Lipworth L, Garidou A et al. (1996) Diet and risk of esophagus cancer by histologic type in a low-risk population. Int J Cancer 68, 300-304.

19. Mayne ST, Risch HA, Dubrow R et al. (2001) Nutrient intake and risk of subtypes of esophageal and gastric cancer. Cancer Epidemiol Biomarkers Prev 10, 1055-1062.

20. Rossi M, Garavello W, Talamini R et al. (2007) Flavonoids and risk of squamous cell esophageal cancer. Int J Cancer 120, 1560-1564.

21. Freedman ND, Park Y, Subar AF et al. (2007) Fruit and vegetable intake and esophageal cancer in a large prospective cohort study. Int J Cancer 121, 2753-2760.

22. Bosetti C, La Vecchia C, Talamini R et al. (2000) Food groups and risk of squamous cell esophageal cancer in northern Italy. Int J Cancer 87, 289-294. 
Appendix Definition of the food groups used in the dietary pattern analysis

\begin{tabular}{ll}
\hline Food groups & \\
\hline Vegetables & Root vegetables, green salads, cauliflower, white cabbage, red cabbage and spinach \\
Tomato & Tomato \\
Fruits & Citrus fruits, apple, pear, banana, plums, apricot, cherry and other fruits \\
Whole grains & Iranian breads (sangak, barbari and taftoon) \\
Refined grains & Rice, noodles, pasta, Iranian bread (lavash) and French bread \\
Legumes & Pea, split peas, bean, lentil, vetch and soya \\
Low-fat dairy & Low-fat milk, low-fat yoghurt and butter milk \\
High-fat dairy & Whole milk, whole yoghurt, cheese, cream and ice cream \\
Fish & Salmon, mackerel, sardines, tuna and other fishes \\
Poultry & Chicken \\
Red meat & Beef, minced meat, liver and mutton \\
Processed meat & Sausage and hamburgers \\
Eggs & Fried eggs, boiled eggs and omelette \\
Sweets & Cookies, chocolate, jam, pastries, tarts and cakes \\
Sugar & Sugar cubes, sugar, tahini halva and candy \\
Nuts & Nuts \\
Tea & Tea \\
Snack & Potato chips and biscuits \\
Fried potatoes & Fried potatoes \\
Cooked potatoes & Boiled potatoes \\
Fruit juice & Juice \\
Pickle & Pickle and pickled cucumbers \\
Solid oil & Solid vegetable oil and solid animal oil \\
Liquid oil & Liquid oil \\
Animal butter & Animal butter \\
Soft drink & Soft drink \\
\hline
\end{tabular}

